\title{
Theory of doped Mott insulators: duality between pairing and magnetism
}

\author{
Qiang-Hua Wang \\ National Laboratory of Solid State Microstructures, \\ Institute for Solid State Physics, Nanjing University, Nanjing 210093, China
}

\begin{abstract}
By bosonizing the electronic t-J model exactly on any two-dimensional (2D) lattices, and integrating out the gauge fluctuations combined to slave particles beyond mean fields, we get a theory in terms of physical Cooper pair and spin condensates. In the sense of mutual Berry phase they turns out to be dual to each other. The mutual-duality is the missing key in the resonant-valance-bond idea[1] to work as a paradigm of doped 2D Mott insulators. We argue that essential aspects of high- $T_{c}$ phenomenology find natural solutions in the theory. We also provide interesting predictions for systems on hexagonal lattices.
\end{abstract}

I. Introduction: Ever since Anderson's proposal that superconductivity in high- $T_{c}$ copper oxides may be a realization of resonant-valence-bond (RVB) physics,[1] solving the t-J model, $[2,3]$ a standard electron model for systems with strong on-site Coulomb repulsion, has been not only a daydream but also a nightmare in the community for more than a decade. The good news is that indeed a lot of high- $T_{c}$ phenomenology may be interpreted in one way or another using the t-J model. The bad news is a consensus to all phenomenology is not reached even just in the underdoped region. It is therefore academically desirable to have a theoretical paradigm (in the infrared limit), just as Luttinger-liquid theory in onedimensional (1D) systems, Landau-Fermi liquid theory for metals and quantum Hall liquid for semiconductor wells under high magnetic fields. On the theory side such a paradigm helps to answer the question: Does t-J model describe high- $T_{c}$ anyway? If it does, what makes high- $T_{c}$ unique? Inspired by the fact that Luttinger liquid theory can be easily brought out by bosonization in $1 \mathrm{D}$ and recent successes of the phase-string theory, [3] in this Letter we tackle the challenge raised by Anderson to generalize the Luttinger liquid to $2 \mathrm{D}$ strongly correlated electron systems. [4] We bosonize the electronic t-J model exactly [see Eqs.(9) and (14)]. The mean field theory integrates spin ordering and pairing thanks to the "all-boson" hamiltonian. We further integrate out gauge fluctuations beyond mean field and obtain a theory in terms of physical Cooper pair and spin magnetic condensates alone [see Eqs.(22), (23), and (25)]. They turn out to be dual to each other in the sense that Cooper pair condensate (spin condensate) sees the classical part of magnetic moments (Cooper pairs) as $2 \pi$-flux bundles.[5]. The mutual-duality between superconductivity and magnetism is clearly what makes doped 2D Mott insulators unique, and is just the missing key in the RVB theory. Because of the intervening magnetism, it seems unnecessary to prepare a quantum spin liquid in parent Mott insulators to adiabatically connect superconductivity. Other far-reaching consequences of the theory are detailed in the closing section after warming up with the notations and technical expositions, which could be skipped for anxious readers.

II. Bosonization of the $\boldsymbol{t}$-J model: The electronic t-J model in the so-called slave-boson representation can be written as

$$
\begin{aligned}
H & =H_{t}+H_{J}-\mu \sum_{i}\left(1-h_{i}^{\dagger} h_{i}\right), \\
H_{t} & =-t \sum_{\langle i j\rangle} K_{i j}^{\dagger} K_{i j}^{h}+\text { h.c. } \\
H_{J} & =-\frac{J}{2} \sum_{\langle i j\rangle} K_{i j}^{\dagger} K_{i j}=-\frac{J}{2} \sum_{\langle i j\rangle} P_{i j}^{\dagger} P_{i j},
\end{aligned}
$$

where

$$
K_{i j}^{h}=h_{i}^{\dagger} h_{j}, K_{i j}=\sum_{\sigma} f_{i \sigma}^{\dagger} f_{j \sigma}, P_{i j}=\sum_{\sigma} \sigma f_{i \sigma} f_{j \bar{\sigma}} \cdot(4)
$$

In the above $h_{i}\left(f_{i \sigma}\right)$ is hardcore holon (fermionic $\sigma$ spinon annihilation operators). We have written $H_{J}$ in the hopping and singlet pairing channels. They help identify the saddle point of the theory in any dimension and for any lattice geometry. The physical Hilbert space is a subspace of the Fock space subject to

$$
h_{i}^{\dagger} h_{i}+\sum_{\sigma} f_{i \sigma}^{\dagger} f_{i \sigma} \equiv 1
$$

The slave-boson hamiltonian is invariant under the local $\mathrm{U}(1)$ gauge transform

$$
f_{i \sigma} \rightarrow f_{i \sigma} \exp \left(i \theta_{i}\right), h_{i} \rightarrow h_{i} \exp \left(i \theta_{i}\right)
$$

This symmetry guarantees the conservation of the local gauge charge $h_{i}^{\dagger} h_{i}+\sum_{\sigma} f_{i \sigma}^{\dagger} f_{i \sigma}$ and the associated current.

While not necessarily unique, we define the following Jordan-Wigner transformation

$$
f_{i \sigma}^{\dagger} \rightarrow N_{\sigma} b_{i \sigma}^{\dagger} \exp \left[i \sigma \sum_{l \neq i} \theta_{i l}\left(n_{l \sigma}-\bar{n}_{\sigma}\right)\right],
$$

were $N_{\sigma}$ is the Klein factor, $b_{i \sigma}$ hardcore boson annihilation operator, $\theta_{i l}$ is a multi-valued function defined by

$$
\theta_{i l}=\operatorname{Im} \ln \left(z_{i}-z_{l}\right), z_{i}=x_{i}+i y_{i},
$$


$n_{l \sigma}=b_{l \sigma}^{\dagger} b_{l \sigma}$ and finally $\bar{n}_{\sigma}$ is the average occupation of $\sigma$-spinons. For systems with anti-ferromagnetic exchange it is reasonable to set $\bar{n}_{\sigma}=(1-x) / 2$ with $x$ the holon concentration. The purpose of including $\bar{n}_{\sigma}$ is to make the gauge field attached to the $b_{\sigma}$-bosons vanish at the mean field level.

By simple substitution and taking into account of the hardcore nature of the bosons as well as the occupation constraint, $H_{J}$ can be rewritten as

$$
\begin{aligned}
H_{J} & =-\frac{J}{2} \sum_{\langle i j\rangle} \mathcal{K}_{i j}^{\dagger} \mathcal{K}_{i j}, \\
\mathcal{K}_{i j} & =\sum_{\sigma} b_{i \sigma}^{\dagger} b_{j \sigma} \exp \left[-i \sigma\left(a_{i j}^{h}+\eta_{i j}\right)\right], \\
a_{i j}^{h} & =\frac{1}{2} \sum_{l \neq i j}\left(\theta_{i l}-\theta_{j l}\right)\left(n_{l}^{h}-x\right), \\
\eta_{i j} & =\frac{1-x}{2}\left(\theta_{i j}-\theta_{j i}\right) .
\end{aligned}
$$

Here $n_{l}^{h}=h_{l}^{\dagger} h_{l}$. The bond phase $\eta_{i, i \pm \delta}=\mp(1-x) \pi / 2$ for $\delta=\hat{x}$ and $\hat{y}$ on square lattices, and for $\delta=\hat{x}$ and $\sqrt{3} \hat{y} / 2 \pm$ $\hat{x} / 2$ on hexagonal lattices. From the above we see that holons appear as $\pi$-flux bundles as viewed by spinnons. This effect is cancelled by the background field due to $x$ at the mean field level, but should be kept beyond the mean field level due to its topological nature. Certainly the above definition of $\mathcal{K}_{i j}$ is unique up to arbitrary spinindependent link phase factors,

$$
\mathcal{K}_{i j} \rightarrow \mathcal{K}_{i j} \exp \left(-i a_{i j}\right)
$$

for any $a_{i j}$, a reflection of the local $\mathrm{U}(1)$ gauge symmetry. We choose not to present the pseudo-pairing operator at this stage. As will be clear, pairing is an inevitable mode in the theory.

Now we can proceed to transform the kinetic part $H_{t}$. By substitution and manipulating in terms of $\mathcal{K}_{i j}$, we get

$$
\begin{aligned}
H_{t} & =-t \sum_{\langle i j\rangle} \mathcal{K}_{i j}^{\dagger} \mathcal{K}_{i j}^{h}+\text { h.c. } \\
\mathcal{K}_{i j}^{h} & =h_{i}^{\dagger} h_{j} \exp \left(-i a_{i j}^{m}\right), \\
a_{i j}^{m} & =\frac{1}{2} \sum_{l \neq i j}\left(\theta_{i l}-\theta_{j l}\right)\left(n_{l \uparrow}-n_{l \downarrow}\right),
\end{aligned}
$$

where we have used the following identity

$$
\sigma\left[n_{l \sigma}+\frac{1}{2}\left(n_{l h}-1\right)\right] \equiv \frac{1}{2}\left(n_{l \uparrow}-n_{l \downarrow}\right) .
$$

Therefore $z$-direction magnetic moments appear as $\pi$-flux bundles as viewed by holons. Again the above definition of $\mathcal{K}^{h}$ is unique up to the same $\mathrm{U}(1)$ gauge transform applied to $\mathcal{K}_{i j}$.

Summarizing, $H=H_{t}+H_{J}-\mu \sum_{i}\left(1-n_{i}^{h}\right)$ with the new forms of $H_{t}$ and $H_{J}$ in Eqs.(9) and (14) discussed above forms another rigorous fully-bosonized representation of the t-J model. The holons and spinnons are mutually frustrated as reflected by the gauge fields $a^{h}$ and $a^{m}$. We note that a similar notion was pointed out in the phase string theory designed particularly for bipartite lattices. [3] The present theory applies for any lattice geometries.

III. Mean field theory: The interacting part of the hamiltonian can be rewritten as $H_{t}+H_{J}=$ $-\frac{J}{2} \sum_{\langle i j\rangle} H_{i j}^{\dagger} H_{i j}+\frac{2 t^{2}}{J} \sum_{\langle i j\rangle} n_{i}^{h} n_{j}^{h}$ with $H_{i j}=\mathcal{K}_{i j}+$ $\frac{2 t}{J} \mathcal{K}_{i j}^{h}$. This suggests the MF effective hamiltonians for holons and spinons, respectively,

$$
\begin{aligned}
& H_{M F}^{h}=-t \sum_{\langle i j\rangle}\left(\chi_{i j}^{*} \mathcal{K}_{i j}^{h}+\text { h.c. }\right)+\mu \sum_{i} n_{i}^{h}, \\
& H_{M F}^{s}=-\frac{J}{2} \sum_{\langle i j\rangle}\left(\chi_{i j}^{*} \mathcal{K}_{i j}+\text { h.c. }\right)-\lambda \sum_{i \sigma} n_{i \sigma},
\end{aligned}
$$

where we suppressed the residual interaction for brevity. Here $\chi_{i j}$ is the mean field hopping amplitude $\chi_{i j}=\left\langle H_{i j}\right\rangle$. The Lagrangian multipliers $\mu$ and $\lambda$ enforces $\left\langle h_{i}^{\dagger} h_{i}\right\rangle=x$ and $\sum_{\sigma}\left\langle b_{i \sigma}^{\dagger} b_{i \sigma}\right\rangle=1-x$, respectively. Let us assume zero average magnetization in the z-direction so that both holons and spinons see zero flux in the MF theory. An obvious mean field solution is $\chi_{i j}=s|\chi|$ with $s=\operatorname{sign}(t)$ so that all three species of bosons can condense to their own non-degenerate ground states: $h_{i}=\sqrt{x}$, and $b_{i \sigma}=$ $\sqrt{\frac{1-x}{2}} \exp \left[-i \sigma s \frac{\mathbf{Q}}{2} \cdot \mathbf{r}_{i}\right]$ with $\mathbf{Q}=\mathbf{Q}_{0}(1-s x)$. Here $\mathbf{Q}_{0}=$ $(\pi, \pi)$ and $2 \pi(1 / 3,1 / \sqrt{3})$ on a square and hexagonal lattice, respectively. By self consistency, $|\chi|=1-x+2|t| x / J$ and $(1-x) \sin [(1-s x / 2) \pi / 3]+2|t| x / J$ on square and hexagonal lattices, respectively. $\mathbf{Q}$ would be the magnetic ordering wave vector as seen from $\left\langle S_{i}^{+}\right\rangle=\left\langle b_{i \uparrow}^{\dagger} b_{i \downarrow}\right\rangle=$ $\frac{1}{2}(1-x) \exp \left(i s \mathbf{Q} \cdot \mathbf{r}_{i}\right)$. The $s$-dependence in $\mathbf{Q}$ on square lattices is trivial because $\mathbf{Q}_{0}(1 \pm x)$ are identical, but is nontrivial on hexagonal lattices. One would also thought of spin pairing $b_{i \uparrow} b_{j \downarrow}$ in the condensates. The bosonized MF state therefore integrates hopping, magnetism and spin pairing. However, we must remind ourselves that the mean field breaks the local $\mathrm{U}(1)$ gauge symmetry. It is a must to integrate out gauge fluctuations beyond mean field theory to talk about physical entities. This is the subject of the gauge theory in the next section.

IV. Integration over gauge fluctuations: The Euclidian action $S=\int L d^{2} x d \tau$ that describes the longwave-length low-energy fluctuations beyond the above MF state is as follows. The Lagrangian density $L=$ $L_{h}+\sum_{\sigma} L_{\sigma}+L_{C S}+i a_{0}$ with $L_{h}, L_{\sigma}$ and $L_{C S}$ describe, respectively, holons, $\sigma$-spinons and the Chern-Simons-like flux-attaching reflecting the mutual frustration between holons and spinons. The holon part is given by

$$
\begin{aligned}
L_{h} & =i\left(\bar{\rho}_{h}+\delta \rho_{h}\right)\left(\phi_{h}^{*} \frac{\partial_{\tau}}{i} \phi_{h}-a_{0}+A_{0}\right)-i \delta \rho_{h} a_{0}^{h}+\frac{u_{h}}{2} \delta \rho_{h}^{2} \\
& +i \mathbf{j}_{h} \cdot\left(\phi_{h}^{*} \frac{\nabla}{i} \phi_{h}-\mathbf{a}-\mathbf{a}^{m}+\mathbf{A}\right)+\frac{1}{2 K_{h}} \mathbf{j}_{h}^{2} .
\end{aligned}
$$


In the above $\bar{\rho}_{h}=x$ is the average holon concentration, $\delta \rho_{h}$ is the holon density fluctuation, $\mathbf{j}_{h}$ is the holon current density, $K_{h} \propto 2 t x \chi$ is the bare holon phase stiffness (this is best seen by integrating out $\mathbf{j}_{h}$ ), $\phi_{h}$ is defined by $h=|h| \phi_{h},\left(a_{0}, \mathbf{a}\right)$ is the auxiliary $\mathrm{U}(1)$ gauge field, $a_{0}^{h}$ is a Lagrangian multiplier for flux attaching to frustrate spinons, $\mathbf{a}^{m}$ is the gauge field due to the frustration from spinons, $\left(A_{0}, \mathbf{A}\right)$ is the physical electro-magnetic (EM) gauge field coupled to the positively-charged holons, and finally $u_{h}$ is an effective short-range repulsion between holons. Similarly, the $\sigma$-spinon part $L_{\sigma}$ is given by

$$
\begin{aligned}
L_{\sigma} & =i\left(\bar{\rho}_{\sigma}+\delta \rho_{\sigma}\right)\left(\phi_{\sigma}^{*} \frac{\partial_{\tau}}{i} \phi_{\sigma}-a_{0}\right)-i \sigma \delta \rho_{\sigma} a_{0}^{m}+\frac{u_{b}}{2} \delta \rho_{\sigma}^{2} \\
& +i \mathbf{j}_{\sigma} \cdot\left(\phi_{\sigma}^{*} \frac{\nabla}{i} \phi_{\sigma}-\mathbf{a}-\sigma \mathbf{a}^{h}\right)+\frac{1}{2 K_{b}} \mathbf{j}_{\sigma}^{2}
\end{aligned}
$$

In the above $a_{0}^{m}$ is a Lagrangian multiplier that attach flux to the z-direction magnetization that frustrates holons, $K_{b} \propto J \chi(1-x) / 2$ is the bare spinon phase stiff- ness, and $\phi_{\sigma}$ is defined by $b_{\sigma}=\left|b_{\sigma}\right| \exp (-i \sigma \mathbf{Q} \cdot r / 2) \phi_{\sigma}$ which describes fluctuations of the $\sigma$-boson beyond the condensate momentum. The last piece $L_{C S}$ is given by

$$
L_{C S}=\frac{i}{\pi} a_{0}^{h}\left(\nabla \times \mathbf{a}^{h}\right)_{z}+\frac{i}{\pi} a_{0}^{m}\left(\nabla \times \mathbf{a}^{m}\right)_{z}
$$

In the above the subscript $z$ means the $z$-component of the object. Let us observe that integration over $a_{0}, a_{0}^{h}$ and $a_{0}^{m}$ using the total action $L$ indeed enforces occupation constraint and attaches $\pi$-flux to holon and magnetization fluctuations, respectively.

Given the above form of the action, we can integrate out the auxiliary $\mathrm{U}(1)$ gauge field $\left(a_{0}, \mathbf{a}\right)$ exactly , [6] yielding $\delta \rho_{h}+\sum_{\sigma} \delta \rho_{\sigma}=0$ and $\mathbf{j}_{h}+\sum_{\sigma} \mathbf{j}_{\sigma}=0$. This enables us to get rid of slave particles in favor of a $\mathrm{U}(1)$ gauge-neutral theory $L=L_{c}+L_{m}+L_{C S}$ as follows.

First, $L_{c}$ describes the charge condensate (CC) of the theory,

$$
\begin{aligned}
& L_{c}=i \bar{\rho}_{c}\left(\phi_{c}^{*} \frac{\partial_{\tau}}{i} \phi_{c}-2 A_{0}\right)+i A_{0}+i \delta \rho_{c}\left(\phi_{c}^{*} \frac{\partial_{\tau}}{i} \phi_{c}+2 a_{0}^{h}-2 A_{0}\right)+i \mathbf{j}_{c} \cdot\left(\phi_{c}^{*} \frac{\nabla}{i} \phi_{c}+2 \mathbf{a}^{m}-2 \mathbf{A}\right)+\frac{u_{c}}{2} \delta \rho_{c}^{2}+\frac{1}{2 K_{c}} \mathbf{j}_{c}^{2}(23) \\
& \bar{\rho}_{c}=\frac{1}{2}(1-x), \phi_{c}=\phi_{\uparrow} \phi_{\downarrow}\left(\phi_{h}^{*}\right)^{2}, \delta \rho_{c}=\frac{1}{2} \sum_{\sigma} \delta \rho_{\sigma}, \mathbf{j}_{c}=\frac{1}{2} \sum_{\sigma} \mathbf{j}_{\sigma}, \frac{1}{K_{c}}=\frac{2}{K_{b}}+\frac{4}{K_{h}}, u_{c}=2 u_{b}+4 u_{h} .
\end{aligned}
$$

Here we have used the identity $\sum_{\sigma} \phi_{\sigma}^{*} \partial_{t} \phi_{\sigma}-2 \phi_{h}^{*} \partial_{t} \phi_{h} \equiv \phi_{c}^{*} \partial_{t} \phi_{c}$. Clearly $\phi_{c}$ is the phase factor of $b_{\uparrow} b_{\downarrow} h^{*} h^{*}$, a spincharge recombined Cooper pair. Moreover, $\bar{\rho}_{c}$ and $\mathbf{j}_{c}$ are just the Cooper pair density and spatial current, respectively, $K_{c}$ is the effective zero-temperature superfluid density, and finally $u_{c}$ is the effective local charge repulsion. At low doping $K_{c} \propto x$. Interestingly, $A_{0}$ in the first two terms of $L_{c}$ simply reminds us that the effective charge density is $1-2 \bar{\rho}_{c}=x$, but the Cooper pairs response to EM field as $2 e$-carriers with pair density $\bar{\rho}_{c}=(1-x) / 2$. This proves that pairing does not imply bounding of holes at all (they are not the normal modes in the theory), a conclusion supported by variational Monte Carlo studies of the t-J+Coulomb model.[7] Moreover, the Cooper pairs also view a localized z-direction magnetic moment as a $2 \pi$-flux bundle as described by $2 \mathbf{a}^{m}$.

Second, $L_{m}$ describes the spin magnetic condensate (MC) of the theory,

$$
\begin{aligned}
& L_{m}=i \delta \rho_{m}\left(\phi_{m}^{*} \frac{\partial_{\tau}}{i} \phi_{m}-2 a_{0}^{m}\right)+\mathbf{j}_{m} \cdot\left(\phi_{m}^{*} \frac{\nabla}{i} \phi_{m}-2 \mathbf{a}^{h}\right)+\frac{u_{m}}{2} \delta \rho_{m}^{2}+\frac{1}{2 K_{m}} \mathbf{j}_{m}^{2}, \\
& \delta \rho_{m}=\frac{1}{2} \sum_{\sigma} \sigma \delta \rho_{\sigma}, \phi_{m}=\phi_{\uparrow} \phi_{\downarrow}^{*}, \mathbf{j}_{m}=\frac{1}{2} \sum_{\sigma} \sigma \mathbf{j}_{\sigma}, K_{m}=\frac{1}{2} K_{b}, u_{m}=2 u_{b} .
\end{aligned}
$$

Clearly, $\delta \rho_{m}, \phi_{m}$ and $\mathbf{j}_{m}$ are the z-direction magnetization $S^{z}$, the phase factor of $S^{-}$and the spin current (beyond the magnetic ordering wave vector $\mathbf{Q}$ ). The gauge field $2 \mathbf{a}^{h}$ describes the effect of Cooper pair density fluctuations on the spin dynamics. A localized Cooper pair would appear as a $2 \pi$-flux bundle to $S^{-}$. On the other hand, MC condensate is not coupled to physical EM gauge fields and is therefore charge-neutral, as would be desirable.

Except for the first two terms in $L_{c}$ (which gives the average density of Cooper pairs and the charge carrier density) the two condensates $\mathrm{CC}$ and $\mathrm{MC}$ are mutually dual to each other. This is the crucial part of the theory that makes a difference to the $1 \mathrm{D}$ counterpart in which spin-charge separation is complete.

V. Discussions: The following discussion at zero temperature relies on the notion of mutual-duality identified above and the self-duality for a separate condensate alone. $[5,8]$ We specialize to the context of high- $T_{c}$ phenomenology, [9] but the basic physics applies to all doped Mott insulators. 1) At zero doping we have a quantum antiferromagnetism described by the $\mathrm{MC}$ alone. The 
gauge field $\mathbf{a}^{h}=0$ since there is no charge fluctuations at all. 2) At low doping $\mathrm{CC}$ is disordered by quantum (or thermal) fluctuations due to the small phase stiffness. The charges are localized and act as vortices to MC by mutual-duality. Depending on whether $\mathrm{MC}$ is of typeI or type-II "superconductor", these charges may either phase separate or form a Wigner crystal, respectively. They correspond to the intermediate state and mixed state in usual superconductors, and would suggest two types of Mott insulators as recently advocated.[10] This is a useful concept to understand the absence or presence of electronic inhomogeneities in different families of high- $T_{c}$ copper oxides.[11] Localized charges can make the spin order glassy. Another interesting possibility is the charge stripe order in cuprates. Since the localized charges appear as vortices to MC, the fact that stripe is an anti-phase boundary to the underlying magnetic order [12] seems very natural. 3) With increasing doping, the charges are more and more mobile and eventually CC shows up above some lower critical doping level. 3a) As we envisioned above, although the doped holes do not pair at all, the Cooper pairs response to EM fields as charge-2e carriers. This determines the correct flux quantum as $h c / 2 e$. 3b) According to the composition rule of superfluid density $K_{c}$ in Eqs.(24), for small $x$ it is dominated by the smaller component $K_{h}$, and is therefore roughly linear in $x$. 4) Consider ordered CC and disordered MC, a superconductor with no spin order. The vortices of MC must condense by self-duality. [5] Let us call this new condensate as $\mathrm{M}^{\prime} \mathrm{C}$. Just as $\mathrm{CC}$ does, $\mathrm{M}^{\prime} \mathrm{C}$ also views localized magnetization $S^{z}$ as $2 \pi$-flux bundles, i.e., the next generation vortex excitations. Therefore $\mathrm{CC}$ and $\mathrm{M}^{\prime} \mathrm{C}$ can happily coexist. This opens up a handful of interesting possibilities. 4a) Vortices under an applied magnetic field in CC may capture local spin magnetic moments, forming non-topological vortices with zero winding number in CC to lower the superflow kinetic energy. Since winding is a necessary condition to the zero energy electron state in a vortex core of d-wave superconductor, [13] non-topological vortices may resolve the puzzle that no vortex-core zero-bias tunnelling conductance peak has ever been observed in cuprates.[14] 4b) A vortex in CC may also capture many spin moments (favorably ordered with staggered polarization), and this would lead to the checkerboard pattern in the local density of states near a vortex core in cuprates.[15] 4c) Moreover, since the captured spins are vortices to $\mathrm{M}^{\prime} \mathrm{C}$ by self-duality, it is favorable to recover MC. This may explain the enhancement of anti-ferromagnetic correlation in the vortex state of cuprates.[16] 4d) The spin excitations $\left(\delta \rho_{m}, \mathbf{j}_{m}\right)$ appear as the "EM" fields to $\mathrm{M}^{\prime} \mathrm{C}$ by self-duality. [5] Such "photons" are gapped up to the plasma frequency of $\mathrm{CC}+\mathrm{M}^{\prime} \mathrm{C}$ state that scales with the physical superfluid density. In view of our starting magnetic vector $\mathbf{Q}$, this gap actually corresponds to the inelastic neutron resonance energy found in cuprates. [17]
The doping dependent incommensurability in $\mathbf{Q}$ is obvious in the theory. 4e) On the other hand, assuming the absence of long-range interactions in either $\mathrm{CC}$ and/or $\mathrm{M}^{\prime} \mathrm{C}$, longitudinal phase fluctuations in the condensates are phonon-like, and via re-fermionization of the emerging low energy field theory[18] they are nothing but the Dirac-like nodal quasi-particles in a d-wave superconductor. 4f) Topologically de-confined but spatially localized spin moments, such as caused by Zn, induces vortices in $\mathrm{CC}$ at zero magnetic field by mutual-duality. Therefore the critical $\mathrm{Zn}$ concentration to kill $\mathrm{CC}$ would be given by $H_{c 2} / \Phi_{0}$. (The spins do not have to point in the same direction.) This seems to agree with the so-called "Swiss cheese" model of Zn in cuprates.[19] More detailed discussions are in progress.

I thank Z. Y. Weng for enlightening discussions and encouragements. This work is supported by NSFC 10204011 and 10021001.

[1] P. W. Anderson, Science 235, 1196 (1987).

[2] See, e.g., M. Grilli, G. Kotliar, Phys. Rev. Lett. 64, 1170 (1990); N. Nagaosa, P. A. Lee, ibid 64, 2450 1990); S.-C. Zhang, Science 275, 1089 (1997).

[3] Z. Y. Weng, D. N. Sheng, Y.-C.Chen, and C.S. Ting, Phys. Rev. B 553894 (1997); Su-Peng Kou and ZhengYu Weng, Phys. Rev. Lett. 90, 157003 (2003).

[4] P. W. Anderson, "Theory of Superconductivity in the High- $T_{c}$ Cuprates", Princeton University Press, 1997.

[5] The concept of duality and useful techniques in another context can be found in, e.g., Dung-Hai Lee, Phys. Rev. Lett. 88, 227003 (2002).

[6] Dung-Hai Lee, Phys. Rev. Lett. 84, 2694 (2000); QiangHua Wang, Jung Hoon Han, and Dung-Hai Lee, Phys. Rev. Lett. 87, 077004 (2001).

[7] Qiang-Hua Wang, Jung Hoon Han, and Dung-Hai Lee, Phys. Rev. B 65, 054501 (2001). See also A. Paramekanti, et al, cond-mat/0305611.

[8] Strictly speaking self-duality is defined only at $T=0$, but mutual-duality applies even at finite temperatures.

[9] Some essential phenomenology was summarized in, e.g., J. Orenstein and A. J. Millis, Science 288, 468 (2000).

[10] Dung-Hai Lee, cond-mat/0208536; cond-mat/0208490.

[11] S. H. Pan, et al, Nature 413, 282 (2002); L. Kristine, et al, Nature 415, 412 (2002).

[12] J. Tranquada, et al, Nature 375, 561 (1995).

[13] Jung Hoon Han, private communications.

[14] I. Maggio-Aprile, et al, Phys. Rev. Lett. 75, 2754 (1995).

[15] J. E. Hoffman, et al, Science 295, 466 (2002).

[16] B. Lake, et al, Science 291, 1759 (2001); Nature 415, 299 (2002).

[17] H. A. Mook, et al, Phys. Rev. Lett. 70, 3490 (1993); P. Dai, et al, ibid 77, 5425; K. Yamada et al, Phys. Rev. B 57, 6165 (1997).

[18] Qiang-Hua Wang, unpublished.

[19] B. Nachumi, et al, Phys. Rev. Lett. 77, 5421 (1996); T. Adachi, et al, cond-mat/0306233. 